\title{
Accounting in the fourth industrial revolution: Exploration of digital currency exchanges using AHP method
}

\author{
Samaneh Sadeghi ${ }^{1 *}$, Jamal Barzegari ${ }^{2}$ \\ Faculty of Economics, Management and Accounting, Yazd University, Yazd, Iran ${ }^{1,2}$ \\ agsh2006@yahoo.com ${ }^{1 *}$,barzegari@yazd.ac.ir ${ }^{2}$
}

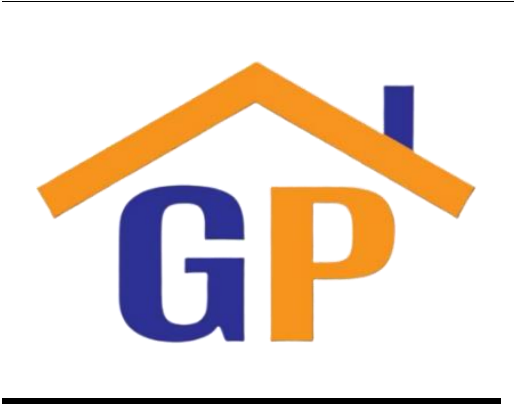

Article History

Received on 9 March 2021

$1^{\text {st }}$ Revisio on 10 June 2021

$2^{\text {nd }}$ Revision on 15 June 2021

$3^{\text {rd }}$ Revision on 5 July 2021

Accepted on 8 July 2021

\begin{abstract}
Purpose: The present study aimed to analyze and select the best exchange in the field of digital currencies with a futuristic perspective.

Research methodology: The research method in this article is to use Analytic Hierarchy Process (AHP) to identify the strengths and weaknesses of each of these exchanges and analyze their coefficients according to the criteria of security, support, commission, number of currency codes, authentication, and trading volume.
\end{abstract}

Results: The most important criteria in choosing the best digital currency exchange are: security, support, commission, number of currency codes, authentication and trading volume.

Limitations: The limitations of this research can be the characteristics of digital exchanges in the field of providing digital currency cryptographic services in accordance with the framework of the Fourth Industrial Revolution.

Contribution: According to the weight of the obtained criteria will be prioritized, which from the results and analysis obtained in this research can be used to invest in the field of currency cryptography and select the top exchange.

Keywords: Fourth industrial revolution, Digital currencies, Blockchain, International exchanges, New accounting

How to cite: Sadeghi, S., \& Barzegari, J. (2020). Accounting in the fourth industrial revolution: Exploration of digital currency exchanges using AHP method. Annals of Management and Organization Research, 2(1), 25-40.

\section{Introduction}

The Fourth Industrial Revolution is the fourth major period since the beginning of the Industrial Revolution. This period is marked by the spread of technologies that reduce or eliminate the distance between the physical, computing, and biological spheres. This course is associated with the emergence of new technologies in several areas of robotics, artificial intelligence, blockchain, nanotechnology, quantum processing, biotechnology, the Internet of Things and automobiles. This revolution transforms the entire system of production, management and governance in every industry and every country (Zahedi \& Khanachah, 2020).

Encrypted (digital) currency has emerged as an alternative to online transactions due to security problems and the high cost of credit and cash cards. Today, millions of people around the world trade in digital currency. Digital currency is the same as electronic money and is used in online stores or even physical stores and transactions between businesses as well as transactions between individuals. With the increase in the price of digital currencies, we will gradually see an increase in the demand for buying and selling and their exchange by digital currency exchanges. Exchanges do the work of securing transactions and, while accepting the risk, also undertake the exchange of currency cryptocurrencies. 
These exchanges are divided into centralized and decentralized. This article examines these exchanges as well as the importance and characteristics of Konus Dex decentralized exchange in the digital currency market (Pichler, Summer, \& Weber, 2020).

The application of blockchain technology in the field of digital currencies, due to the promotion of key indicators such as improving security in the context of non-forgery, unchangeable information, tracking, decentralization and transparency, makes the application of this technology in digital currencies and service companies in this field more important. To be placed (Foroglou \& Tsilidou, 2015). Readiness to accept this technology involves accepting a set of managerial and organizational criteria. Therefore, the present study was conducted to aim for future investment research in digital currencies. The research method in this article is to use Analytic Hierarchy Process (AHP) to identify the strengths and weaknesses of each of these exchanges and analyze their coefficients according to the criteria of security, support, commission, number of currency codes, authentication and trading volume. Finally, according to the weight of the obtained criteria, they will be prioritized. The results and analysis obtained in this research can be used to invest in the field of currency cryptography and select the top exchange.

\section{Literature review Digital exchange}

After hearing the name of an exchange office, the first thing that comes to mind is buying and selling and converting currencies into each other. About currency cryptography, this is the first and most important service that cryptocurrency exchanges offer. Exchanges also do the work of securing transactions and, while accepting risk, also undertake currency exchange exchanges. In recent years, exchange offices have also provided various opportunities to buy through digital currency and pay employees with cryptocurrencies. This way, freelancers can get paid for their services to Bitcoin with other cryptocurrencies. In the digital currency market, exchanges are divided into the following two categories (Anderson, Shumailov, Ahmed, \& Rietmann, 2019).

\section{Blockchain}

Blockchain is an undeniable innovative technology that has revolutionized the world. This technology has a longer lifespan than phenomena such as digital currency, currency cryptography, or smart contracts. In its simplest form, Blockchain is a timed series of unstable data records managed by a group of computers that do not belong to a particular unit. These secure data blocks are chained together using cryptographic principles containing only valid transactions, thus preventing fraudulent transactions and recurring attacks. The rate obtained consists of numbers and letters that bear no resemblance to the original data and are known as hashes. The hash functions in the blockchain generate a fixed-length output in exchange for receiving the desired input and applying some mathematical algorithms to it, which is used to verify and ensure that the files are not tampered with and maintain their integrity. The figure below shows how to connect the blockchain chain and hash functions (Brunton, 2020).

China's blockchain technology has created the backbone of the Internet by making it possible to distribute digital information without copying it. Designed initially for bitcoin digital money, the technology community is now finding other potential uses for the technology. Blockchain is an indestructible digital general ledger of economic transactions that can be used to record financial transactions and record almost any valuable asset (Mainelli \& Smith, 2015). Blockchain is a decentralized technology. Everything that happens on it is the result of the network functioning as a whole. This feature is used a lot. By creating a new way of looking at transactions, some aspects of traditional trading will lose their relevance. For example, stock exchange transactions can be placed in the Chinese block at the same time, or a variety of audits such as land registration can be made completely public (Di Silvestre et al., 2020).

\section{Centralized and decentralized exchanges}

Centralized exchanges, as a third party, monitor the relationship between buyers and sellers of digital currency. Some centralized exchanges are compared to banks for their mission of securing and 
overseeing digital assets. However, transactions made in centralized exchanges have features that are considered a disadvantage for major investors and people who intend to trade and transfer money. Among these features are blocking money in the exchange account, intermediaries' presence, and timeconsuming (Aaron, Rivadeneyra, \& Sohal, 2017). Also, with the increase in the value of the cryptocurrency market and the increase in the cryptocurrency price, hackers' attention has been drawn to this market, and hackers have attacked several centralized cryptocurrency exchanges. Decentralized exchanges were a way to combat this new form of hacking. These exchanges are very resistant to hacking and have significantly improved the security of users' digital assets by eliminating the storage of private key information on exchange servers. Previously, centralized exchanges stored users' private keys on their servers, allowing hackers to steal users' property by hacking the system and accessing this information(Howell, Niessner, \& Yermack, 2020).

According to the World Economic Forum, repetitive jobs (i.e., rule-based, Coin -minded, and automated) are more than $95 \%$ likely to be replaced by intelligent systems, machines, or robots. Jobs include cashiers, bookkeepers, accountants, and the like, which means that the nature of future jobs will definitely change as a result of the Fourth Industrial Revolution. The importance of this issue is so high that the main focus of the Davos Summit (32nd World Economic Forum) in 2019 was globalization in the era of the Industrial Revolution and the participants of this summit (including heads of state and 1700 influential private sector executives in the world and 900 leaders of NGOs and labor organizations, religious groups, media activists, academics and social entrepreneurs) discussed and reported on creating a global framework that meets the needs of the present or the era of the Fourth Industrial Revolution. . According to research, the majority focus on the awareness of actors in the industry (ie senior managers and accountants and financial managers) about the Fourth Industrial Revolution.

In decentralized exchanges, all transactions are done automatically through smart contracts, which has significantly increased the security of transactions. In this way, digital assets will not be stored and blocked in any way and hackers will be deprived of properties (Savelyev, 2017). Konus Dex Decentralized Exchange offers a decentralized currency conversion service, allowing users to exchange peer-to-peer currency most conveniently and securely possible through China Blockchain technology and directly convert currency codes with each other or with Fiat currency. Konus Dex Currency Exchange Service allows users to manage their currencies without a third party directly. These transactions will guarantee their security and accuracy. On the other hand, because users do not need to present their digital assets to exchange in decentralized currency conversion, the risk of hacking and stealing assets will be reduced to a minimum. In this article, decentralized exchanges and their role in the cryptocurrency market and the features of Konus Dex decentralized exchanges will be examined.

In recent years, as people have become more interested in digital currencies, digital currency exchanges are also trying to provide better services to traders. One of the main questions of digital currency users is the difference between centralized and decentralized exchanges. In the following, we will examine the benefits of each of these exchanges.

\section{Centralized exchanges}

Centralized exchanges are used for trading in the digital currency market. These types of exchanges have the ability to convert Fiat currencies (common currencies) to digital currencies and vice versa. They can be used to convert two digital currencies to each other.

In the following, we will briefly review some of the centralized exchanges:

\section{Bitrex centralized exchange}

Bitrex is a centralized digital currency exchange where a very high volume of transactions is done daily. The main reputation of this exchange is due to its very high security due to two-step authentication. It is also possible for users to trade more than 190 digital currencies in Bitrex Exchange. 
Operated by iFinx, this exchange is one of the oldest exchanges in the digital currency market. The exchange was established in 2012 and is located in Hong Kong. The most prominent feature of this exchange that has made it popular among users is the support of the most important digital currencies. Some of these currencies are Bitcoin, Atrium, Ripple, and others.

\section{POLONIEX centralized exchange}

This exchange can be called one of the safest and most popular exchange offices in the United States. The exchange was established in 2014 in the United States. One of the salient features of this exchange is that the fee for charging and withdrawing accounts in the Politex exchange is zero. Moreover, while centralized exchanges seem to cover all digital currency exchanges, other types of exchanges offer many security and services to their users.

\section{Decentralized exchanges}

Decentralized exchanges are a new generation of trading platforms. In these types of exchanges, a completely safe and reliable environment is provided for users and there is no need for a third party to do business. In addition, the structure of decentralized platforms is designed in such a way that all transactions are done automatically through smart contracts, which has significantly increased the security of transactions. This way, digital assets will not be stored or blocked in any way, and hackers will be deprived of property. In the following, we will introduce some of these decentralized exchanges (Di Silvestre et al., 2020).

\section{Decentralized Bainance Exchange}

Bytense can be considered one of the leading companies in the field of digital currencies. This exchange operates in two centralized and decentralized ways. The company's goal is to set up Bitcoin and other digital currencies in a decentralized manner, providing better terms and facilities and a reliable financial environment for exchanging digital currencies. They believe that individuals have more power by removing the controlling authority because they are given complete control of capital (Dell'Erba, 2019).

\section{Decentralized Bitsquare Exchange}

Bitsquare Decentralized Exchange, formerly known as Bitsquare, can be introduced as the first active exchange of Bitcoin and other cryptocurrencies in a decentralized manner since 2014. This exchange is an open-source program that allows people to buy and sell digital currencies without the need for registration or any approval of the central institution. One of the outstanding features of this exchange is wallets equipped with multiple signatures. Multiple signatures mean that after the digital currency trading order is matched, the trader obtains three endorsements from the seller, buyer, and intermediary.

\section{Conus Dex Decentralized Exchange}

Conus Dex is managed by Counos OU. This company is registered in Estonia with the registration number and legal license code 14673576. It has a license to operate in the field of currency exchange and Fiat currency (Rials, dollars, etc.). This exchange uses a smart contract to hold assets. One of the most important features of this decentralized exchange is introducing a new dual feature for fast trading or digital currency trading. The task of this dual system is, in fact, to adapt and sort digital currency exchanges. In this service, users will be able to guarantee transactions related to the exchange of currencies with each other in the presence of a third party or supervisor. The Conus Dex platform provides a highly secure algorithm for converting currencies by providing a private three-way key to trading and third parties. By registering at least two parts of the private key, the transaction will be finalized. In fact, the final consent of the buyer and seller or the guarantee of a transaction by either party with a third party or supervisor will confirm the exchange of currency. Given the high potential of this service in use as a secure decentralized exchange, we will continue to examine its features.

The first and most prominent feature is that unauthorized persons in transactions of digital currencies to other digital currencies (crypto to crypto) can conduct their transactions without intermediaries. Both parties' information is protected, and the intermediary is eliminated. However, in order to conduct digital currency transactions with fiat money or the common currency and vice versa, users of the 
decentralized exchange Konus Dex must authenticate in three steps (1- phone number, 2- personal or company details, 3- bank details) and upload documents (which may include a driver's license, passport, ID card, face image, and bank certificate). Of course, it should be noted that these measures are to maintain security for users and prevent any abuse and fraud in order to create a safe environment for users who are concerned about the security of their capital in using this new technology. The second distinctive feature of this type of decentralized exchange is its high security, which is a space for hacking in a world where investors and traders have transferred a large part of their capital to the cryptocurrency market, which has increased liquidity in digital markets. It has limited the millions of dollars traded in them and has provided high security against cyber attacks and hacker intrusion. Furthermore, their third prominent feature is the increase in transaction speed (Mokhtarian \& Lindgren, 2018).

\section{The advantage of Conus Dex Decentralized Exchange over other exchanges}

Cots Dex Decentralized Exchange has created special conditions for its users compared to other decentralized exchanges. Using blockchain technology and cryptocurrencies, the decentralized exchange office of Kutus Dex has created a space for users. Each person and company can act as an exchange office on their own, and any business transactions, sales, money transfers, etc., currency exchange to Fiat money (Common) and vice versa and convert currency codes to each other without intermediaries and with the assurance of non-abuse. This exchange has the following features for the security of its users: First, the decentralized exchange Konus Dex has an official license registered in the European Union, along with offices and legal entities, and registered and official contact numbers with valid links. Second, the capital of individuals is not made available to the exchange or other persons. According to the people's password in the blockchain system, there are three signatures, and each signature is according to the password of the people in the blockchain system. The screw system gives this password to use this wallet to do only that transaction, and only those who have this password have access to this wallet. (Which includes the buyer and seller and the official and legal representative) (Batent, Taheri, Borbor, \& Nasri, 2020).

\section{How to trade through Conus Dex Decentralized Exchange}

To carry out any interpersonal transactions, especially people who do not know each other and intend to trade and transfer money, multi-signature wallets are used to create security in the transaction and prevent any fraud in the decentralized exchange of Konus Dex. The multi-signature wallet in which the currency is stored has three signatures: the signature of the buyer, the seller, and the legal representative (screw agent, legal and official representatives) to ensure that the parties make the transfer and that at least two signatures are required to be released. For example, suppose transactions are in the field of buying or selling digital currency in exchange for currency with prices. In that case, people can first register their offer in the offer or request section, and according to their offer, people will meet the conditions accordingly. The request will be introduced in the market in this exchange. With the final selection and in order to finalize the first purchase, the exchange will ask the seller to store digital currency in the wallet with several signatures, but this does not mean that the stored currency will reach the seller or buyer. Then to release, it requires at least two signatures from these three sellers (purchased by the screw representative). It should be noted that there are three legal representatives of this company (Screw Agent) in this exchange, which includes a law firm with an official lawyer in the European Union, an official lawyer in Switzerland and the representative of the self-centered exchange Konus Dex, which is officially licensed. Each of the representatives has its own commissions that people can choose at will. Despite these escrow agents who monitor transactions and have one of the proprietary passwords for multiple wallets, people can be sure that they will definitely get the capital they want by remittance or currency storage. At this stage, after storing the currency code in the wallet, the buyer will make a few signatures of the desired remittance and scan the receipt and upload it in the system, which has a time of 22 hours to confirm the financial receipt, after which if there is a problem and receive Failure to do so, each party to the transaction has the right to object, at which time the screw representative after reviewing the documents and who is entitled, given that the currency code is released with two signatures, can assign the currency code to the right person. Slowly without suffering any damage. Another type of transaction is digital currency to digital currency transactions. Both parties to the transaction must store their currencies in the wallet within a maximum of ten hours, which will be done by the representative and easily transferred. 


\section{Introducing the best exchange websites in the world}

\section{Coinbase Digital Currency Exchange}

Coinbase is a digital currency exchange owned by the United States, which is genuinely one of the largest digital currency exchanges in the world (Ding et al., 2015). In addition to an Exchange website, Quinn Base also has a wallet where digital money can be stored. CoinBase supports Bitcoin, Atrium, BitcoinCash and LightCoin, and other digital currencies in 32 countries. Kevin Bass is primarily focused on four major digital currencies:

- Bitcoin (BTC)

- Bitcoin Cache (BCH)

- LightCoin (LTC)

- Etherium (ETH)

Nevertheless, it also supports other digital currencies such as XRP, EOS, XLM, ETC, ZEC, ZRX, REP, BAT, LINK, DAI, USDC. Users can use it with their bank account, credit card, PayPal, or Other payment methods that provide digital currency. The platform has 10 million users and exchanges $\$ 50$ billion in digital money. According to Alving et al. (1984), the security level of Quinbase B is considered one of the most important and reliable exchanges in the world. It is also an old exchange, as it has been operating since 2012. Despite its limited coin support, it sometimes pushes it out of the cycle of choosing the best digital currency exchange. This American exchange has chosen variable wages as its strength. This has led to the exchange attracting more than 10 million users (Plesner \& Husted, 2020). Strengths:

- Security

- Variable fees weak points:

- Restrictions on tradable coins Kevin Bass has a high reputation and credibility among traders.

Kevin Bass charges $2 \%$ on bank transfers and $2 \%$ on credit card purchases. The transaction fee in Coinbase also varies based on the volume of transactions. The number of digital currencies that can be bought and sold in Coinbase is limited. Use Quinn Base digital currency exchange if: people are new users. Security and credibility are important. People want to buy from credit cards or bank account in dollars. People live in a country supported by Kevin Bass (USA, Europe, Singapore, Australia, etc.). This exchange does not provide services to Iranian citizens.

\section{Bainance Digital Currency Exchange}

Exchange Bainance website allows users to buy and sell more than 200 digital currencies and perform these transactions with low fees. This site provides exchanges of base currencies including Bitcoin, Atrium, LightCoin and Binance Coin. Bainance was founded in Hong Kong. Currently, most transactions are done in this exchange. While having a second level approval in this exchange allows up to 100 bitcoins to be withdrawn per day, having a first level approval allows only withdrawing less than two bitcoins per day (Go et al., 2014).

Bainance Digital Currency Exchange, although short-lived, has snowballed. The public exchange started in China but has now moved to Malta in the European Union. Bainance is known for its cryptoto-crypto transactions. This exchange is one of the largest exchanges in the world today. About 300 different coins can be traded in this exchange, and this variety of choices, plus low fees when making transactions, has made this exchange one of the largest digital currency exchanges. Of course, do not forget that people have to pay their desired digital currency price with another digital currency when buying from this site. In principle, this exchange is used to convert currency codes (Allayannis, Ihrig, \& Weston, 2001).

Strengths: • Multiple tradable digital currencies $\bullet$ Low commission weak points: • Do not support Fiat currencies when buying - Bainance is weak in response, but the company's customer service system supports 15 world languages, which supports the exchange's claim to be international. Benefits Advantages: - A wide range of digital currencies can be traded in it. Binance fees are very reasonable and allow the user to register immediately. - Limited use of Bainance services does not require authentication. (The limitation of this issue is the withdrawal of up to two bits on a daily basis. To 
increase the withdrawal limit, it is necessary to send an ID card and a selfie photo to Bainance.) Bainance saves $10 \%$ of its commissions in the users' asset insurance fund to compensate in case of any problem, including hacking of this platform in 2019. - The possibility of exchanging Fiat with the currency code in Bainance is gradually being created. The following three features have led many to consider Bainance as one of the best digital currency exchange options. $\bullet$ High speed $\bullet$ Ease of use $\bullet$ Low fees.

Bainance has prohibited Iranian users from working in its system in terms of using its services. However, considering that people will not need to authenticate until they want to withdraw more than two bitcoins per day from this exchange, as long as people do not connect to this exchange with Iran IP, people can avoid the update. The problem in this exchange is sure. However, at present, if people's account is found to belong to citizens of prohibited countries, including Iran, they will be given the opportunity to withdraw property from Bainance. Use Bainance Digital Currency Exchange if: Nonauthentication up to a certain withdrawal limit is important to people. Low trading costs (commission, market depth, spreads) are a priority for people. People need to trade a wide range of digital currencies. People are looking for a credible and up-to-date sketch.

Bainance Exchange charges a fee starting at 0.1 percent for each transaction under $\$ 50$ for each transaction, and the lower the transaction amount, the lower the fee. Bainance uses the same method to earn money. Of course, do not forget that if one of the parties to the transaction uses the BNB coin, which is a coin for Bainance, this fee will be reduced by up to 25\%. On May 7, 2019, Bainance announced that hackers had been able to exploit a security breach and steal 7,000 bitcoins worth $\$ 40$ million at that time. Zhao, one of the founders of the exchange, regretted that the hackers had used various methods to steal. Hackers have been able to bypass the security barriers of this exchange by resorting to phishing, counterfeiting, viruses and other attacks. Bainance had banned cryptocurrencies for some time, but the trading process resumed after some controversy. According to Irresberger et al $\underline{(2020)}$, the security level is considered binance $c+$. Bainance Exchange announced that it will compensate the damage. With all these ups and downs, trading peaked again in Bainance, and Bainance is now recognized as one of the largest exchanges in the world. Zawo announced in early 2020 that the number of new registered users at the exchange had increased dramatically, which is good news for the company. Bainance later announced that it had returned the hacked amounts to users' accounts.

\section{Bitrex Digital Currency Exchange}

Bitrix is another American exchange that allows users to trade more than 250 currency pairs. Therefore, many cryptocurrencies will be available to the user on one platform. Bitrex Exchange was officially launched in 2014 by Bill Shihara, Ricky Lai, Rami Kovacs, and Ryan Huntz in order to gain people's trust in digital currencies. The exchange is located in Seattle, Washington, and its founding team has more than 50 years of experience in security and development for companies such as Microsoft, Amazon, Qualiz and BlackBerry. Security is key for Bitrex Exchange. Bitrex Exchange is for trading a currency code with another currency code. In this exchange people can only trade in encrypted currency. This means that you cannot buy cryptocurrencies with government-approved currencies, such as the US dollar, the British pound, and the Chinese yuan. In Bitterx, trading pairs are two common currencies for which a trading rate is offered. Bitcoin and Tetra are the most popular trading pairs, and Bitcoin alone has 283 pairs of information in the Bitrex exchange. The dollar equivalent of Bitrex Exchange transactions reached more than \$248 million in 2018 and was among the top 15 exchange offices in the world this year. One of the good aspects of Bitrex Exchange is having a fixed fee of $2 \%$ for low value transactions. This amount of commission in some transactions is slightly higher than the average commission received by other exchanges. However, for beginners it makes it easier to calculate the actual amount of income and purchases accurately. The authentication process at Bitrex Exchange is done through a service called Jumio. The Jumio service can authenticate more than 100 million people in more than two thousand countries. In this service, people only need to give their name, address and date of birth, which means that all traders from all over the world can use this exchange. The Bitrex trading system is based on elastic calculations that are designed to process very high volume of transactions. To be exchanged for US dollars. Another downside of Bitrex Exchange is the late response 
and poor communication with the exchange's customers, as some users have been waiting for weeks for their authentication response.

If security is important, Bitrex can be considered the best digital currency exchange in this regard. If people ask what is the best digital currency exchange in terms of speed, Bitrex will still be the answer. Beatrice supports about 250 different coins. Add to that the easy user interface. The only downside is that Fiat currencies are not accepted when registering transactions. In other words, like Bainance, it only supports currency codes. According to Irresberger et al (2020), the security level of Bitrex c is considered to be appropriate. Bitrex is very committed to providing high security and proudly claims to use several layers of protection and the best security technologies and techniques to protect deposits as well as customer information. The platform was also able to reach $99.95 \%$ uptime, which indicates the reliability of this exchange engine. Strengths: - High speed $\bullet$ high security Easy user interface • Supports 250 types of digital currencies weak points: - Can only be used to convert currencies to each other Of course, Bitrex Exchange was very notorious among Iranian users because last year all assets discovered from Iranian users were blocked and no legal documents were provided for it.

\section{New exchange engine}

In February 2019, Bitrix announced to its customers that it was upgrading its trading engine. The purpose of this update was to increase the speed of the platform, its scalability, as well as to build a smooth path for new features in the future. Following this update, the speed of updating the orders section has increased and also, the execution of general orders has become faster and smoother. In fact, commands are now executed 20 times faster than before. Liquidity The amount of liquidity in this exchange is appropriate, but there is still a long way to go to reach the top 10 digital exchanges in the world in terms of liquidity. In February 2019, Bitrex daily trading volume was estimated at nearly \$13 million. In June of that year, it increased nearly fivefold to \$55 million. These figures have fallen sharply in the current era and the global crisis of the Coronavirus, and in the latest statistics for March 2020 , it was only able to reach a trading volume of $\$ 25.3$ million. It can be concluded that the trading volume on this platform is experiencing more frequent and larger changes than other platforms. Bitrex Trading Fees Beatrix is one of the platforms that does not consider separate and different fees for buyers and sellers. Such platforms are commonly referred to as flat fees. Bitrex considers a fixed transaction fee equal to $0.2 \%$ of the trading volume. This seems like an attractive trading fee model for investors who prefer to order based on the orders section's orders. Bitrex fixed prices are almost in line with the industry average. The global average of these fees over a long period of time has been approximately $0.25 \%$. Today, however, we see more and more declines towards lower wages, such as $0.10 \%$ to $0.15 \%$. In the short term, we may see the average wage of $0.10 \%$ dominate this industry.

\section{OKEx Digital Currency}

Exchange Originally established in 2014 in China, OKX is an exchange with more than 145 coins and a high volume of 24-hour trading, which also launched the OKB coin. Variable fees are another point of working with this exchange. Unlike most exchanges that have restricted Iranian users, this exchange restricts American users and does not provide services to them(Picard et al., 2015). Strengths: • Supports 145 different coins $\bullet$ Variable fees OKEx Exchange is one of the leading digital currency exchanges in the world and provides blockchain technology to provide advanced financial services to traders worldwide. According to Irresberger et al. (2020), it is considered the security level of OKEX-B. The OKEx exchange provides traders with access to hundreds of currency codes and futures trading pairs. It is also one of the top digital currency exchanges in terms of trading volume, serving millions of users in more than 100 countries. OKEx Exchange offers traditional trading pairs along with derivatives and other advanced trading facilities. OKEx Exchange offers various trading tools, including spot trades, margin trades, futures contracts, permanent swaps, and options trade. OKEx service fees are very suitable for such a diverse exchange. The OKEx exchange charges a $0.1 \%$ commission for all token-totoken transactions and $0.15 \%$ for a vector order (Picard et al., 2015). 


\section{Bybit Digital Currency}

Exchange Bybit was established in 2018 in Singapore and had offices in Hong Kong and Taiwan. This exchange operates under the supervision of Bayte Fintech Limited; The company is registered in the British Virgin Islands, where it hosts many organizations and experts in the Chinese blockchain and its financial markets. Bybit is not a regular exchange but a digital currency derivatives exchange. The main focus of this exchange is bitcoin/dollar and atrium/dollar transactions, but it also supports a number of other currencies (Salganik, 2019). Extensive support Bybit supports Bitcoin, Atrium, EOS and Ripple currencies. This is one of the unique advantages of Bybit that these 4 major currencies can be traded together in one exchange. Each of these currencies can be traded as derivatives and leveraged by long orders or shorts. The exchange supports BTC/USD, BTC/USDT, ETH/USD, XRP/USD, EOS/USD currency pairs. This means that users can trade based on the mentioned currency pairs.

In terms of order processing speed, Bybit is one of the new exchanges today. This high speed helps the Baybite network to operate smoothly during hard and turbulent times, without overload. This is a problem that BitMax has faced most of the time; Delays or the inability of the network to register or cancel orders in a short time can be risky. Customer services One of the great advantages of Baybite is its active and powerful customer service (and in different languages). People can contact support via their dashboard home page. They can also connect directly with Baybite administrators on Twitter. Security and anonymity Multi-Signature and Air-gapped wallets for security, and the possibility of anonymity for traders Lack of strict customer identification policy or (KYC) are features that draw the attention of Bybit users concerned about their security when trading.

\section{Research Methodology}

Futurology is the discovery and analysis of factors influencing the future to create a favourable future. Today, change is happening at a faster rate. Technological changes and subsequent changes in other aspects of life, increasing interdependence of countries and nations, decentralization of existing societies and institutions accelerated by the spread of information technology, increasing tendency to globalize while preserving national, ethnic and cultural characteristics and many Other factors necessitate a better understanding of "change" and the "future" for governments, businesses, organizations, and people. Therefore, due to the high importance of investing in new fields, especially cryptocurrencies, it causes us to have a different and practical view of this phenomenon scientifically, and through proper analysis of this technology and related service companies; Manage the risks involved as much as possible.

The method of hierarchical analysis is one of the most powerful multi-criteria decision-making techniques presented in 1980 by Saati, a professor at the University of Pittsburgh, and was welcomed by the scientific community despite some criticisms. This approach, reflecting natural behavior and human thinking, enables decision-makers to present the interaction between different factors in complex and unstructured situations. This technique facilitates decision-making by organizing emotions, perceptions, estimates, judgments and identifies the forces influencing the decision. The hierarchical analysis process is one of the most comprehensive systems designed for decision making with multiple criteria. The technique allows the problem to be formulated hierarchically and allows different quantitative and qualitative criteria to be considered in the problem. This process incorporates different options in decision-making and allows the analysis of sensitivity to criteria. In addition, it is based on pairwise comparisons, which facilitate judgments and calculations. It also shows the degree of consistency and incompatibility of the decision, which is one of the advantages of this technique in multi-criteria decision-making. In addition, it has a strong theoretical basis and is based on the final principles, which we will explain in the following. By analyzing difficult and complex problems, the hierarchical analysis process turns them into a simple form and solves them. In fact, hierarchical analysis is a way to help decision-makers to prioritize and classify their goals and strategies in a complex, unstructured and non-transparent environment (Hakimi-Asiabar, Ghodsipour, Seifi, Kerachian, \& O'Brien, 2008). 
In this method, the decision problem is divided into different levels of objectives, criteria, sub-criteria, and options. The decision-maker can easily be careful in the smallest decision. To build a decisionmaking model, criteria are set at the highest level of the target, at the intermediate level or levels of criteria, and the lowest level. In this method, the decision problem is divided into different objectives, criteria, sub-criteria, and options. The decision-maker can easily be careful in the smallest decision. To build a decision-making model, criteria are set at the highest level of the target, at the intermediate level or levels of criteria, and at the lowest level. Purpose: The main research question or problem to be solved is called the purpose. The goal is the highest level of the hierarchical chart and has only one parameter, the selection of which is the task of the highest project decision. Criteria: Criteria that include the goal and the constructor are called criteria. Criteria are, in fact, the benchmark or means of measuring it. The more criteria the criteria cover and the more expressive the goal, the more likely it is that the result will be more accurate. Criteria are the second level of the hierarchical chart after the target. At this level, the required number can be drawn and adjusted horizontally as needed. Criteria can be divided into sub-criteria and sub-criteria can be divided into subsequent criteria. This situation can increase up to $\mathrm{n}$ sub-criteria vertical and horizontal depending on the necessity (Khaleghi Moghadam \& Khalegh, 2009).

The method of hierarchical analysis is one of the most powerful multi-criteria decision-making techniques presented in 1980 by $\underline{\text { Saati }}$, a professor at the University of Pittsburgh, and was welcomed by the scientific community despite some criticisms. This approach, which reflects natural behavior and human thinking, enables decision-makers to present the interaction between different factors in complex and unstructured situations. This technique facilitates decision-making by organizing emotions, perceptions, estimates, judgments, and identifies the forces influencing the decision(Saati, Memariani, \& Jahanshahloo, 2002). The hierarchical analysis process is one of the most comprehensive systems designed for decision making with multiple criteria. The technique allows the problem to be formulated hierarchically and allows different quantitative and qualitative criteria to be considered in the problem. This process incorporates different options in decision-making and allows the analysis of sensitivity to criteria. In addition, it is based on pairwise comparisons, which facilitate judgments and calculations. It also shows the degree of consistency and incompatibility of the decision, which is one of the advantages of this technique in multi-criteria decision making. In addition, it has a strong theoretical basis and is based on the final principles, which we will explain in the following. The hierarchical analysis process, by analyzing difficult and complex problems, turns it into a simple form and solves them. In fact, hierarchical analysis is a way to help decision makers prioritize and categorize their goals and strategies in a complex, unstructured and non-transparent environment.

\section{Findings}

The options are actually the purpose and destination of the target in the hierarchical diagram and the target response is obtained from the options drawn. The options are the last level of the hierarchical chart and depend on how the hierarchical analysis method is used. In cases where this technique is used to select or prioritize, the researcher usually determines the options because he determines which options should be selected or which options should be prioritized. The following figures show a simple hierarchical structure and a hierarchical structure with the following criteria (Tahriri. F, 2008).

\section{Customer support}

Many new traders value customer service and exchange support. Interestingly, while Bainance support is limited to email and social media interaction, Bybit has gone a step further and is responding to users' needs around the clock through online chat, email and even telegram. 
Table 1. Weight of basic criteria of digital exchanges

\begin{tabular}{|c|c|c|c|c|c|c|}
\hline & $\begin{array}{c}\text { Trading } \\
\text { volume }\end{array}$ & Authentication & $\begin{array}{c}\text { Number of } \\
\text { currency } \\
\text { codes }\end{array}$ & Wage & Support & Security \\
\hline Security & $1 / 2$ & 4 & 3 & $1 / 4$ & 2 & $\mathbf{1}$ \\
\hline Support & $1 / 3$ & 3 & 2 & $1 / 5$ & 1 & $\mathbf{1 / 2}$ \\
\hline Wage & 2 & 6 & 5 & 1 & 5 & $\mathbf{4}$ \\
\hline $\begin{array}{c}\text { Number of } \\
\text { currency } \\
\text { codes }\end{array}$ & $1 / 4$ & 2 & 1 & $1 / 5$ & $1 / 2$ & $\mathbf{1 / 3}$ \\
\hline $\begin{array}{c}\text { Authentication } \\
\text { Turnover }\end{array}$ & $1 / 5$ & 1 & $1 / 2$ & $1 / 6$ & $1 / 3$ & $\mathbf{1 / 4}$ \\
\hline Total & 4.28 & 21 & 15.5 & 2.31 & 11.83 & $\mathbf{8 . 0 8}$ \\
\hline
\end{tabular}

Table 2. Paired comparison matrix

\begin{tabular}{|c|c|c|c|c|c|}
\hline $\begin{array}{c}\text { Intermediate } \\
\text { values }\end{array}$ & $\begin{array}{c}\text { Extraordinary } \\
\text { importance }\end{array}$ & $\begin{array}{c}\text { Very strong } \\
\text { importance }\end{array}$ & $\begin{array}{c}\text { Strong } \\
\text { importance }\end{array}$ & $\begin{array}{c}\text { Medium } \\
\text { importance }\end{array}$ & $\begin{array}{c}\text { Equal } \\
\text { importance }\end{array}$ \\
\hline $2 ، 466$ & 9 & 7 & 5 & 3 & 1 \\
\hline
\end{tabular}

Table 3. The product of the weight of the criteria in pairwise comparison to check compatibility

\begin{tabular}{|l|l|l|l|l|l|l|l|}
\hline 0.149 & 0.92 & 0.12 & 0.16 & 0.19 & 0.10 & 0.19 & 0.15 \\
\hline 0.096 & 0.58 & 0.08 & 0.12 & 0.13 & 0.08 & 0.10 & 0.07 \\
\hline 0.403 & 2.53 & 0.48 & 0.25 & 0.32 & 0.40 & 0.48 & 0.60 \\
\hline 0.064 & 0.38 & 0.06 & 0.08 & 0.06 & 0.08 & 0.05 & 0.05 \\
\hline 0.041 & 0.26 & 0.05 & 0.04 & 0.03 & 0.07 & 0.03 & 0.04 \\
\hline 0.24 & 1.49 & 0.24 & 0.21 & 0.26 & 0.20 & 0.29 & 0.30 \\
\hline
\end{tabular}

\section{Weight criteria}

So our $\lambda \max$ is equal to multiplying the weighted sum values by the weight of the criteria and then adding them and dividing by 6 , which is equal to: $6.162192 \lambda \max -n / n-1=0.03243$ In the above formula, $\mathrm{n}$ is the number of criteria, which is 6 here. The random index for $\mathrm{n}=6$ is 1.24 , so dividing 0.03243 by 1.24 gives the compatibility rate equal to 0.02615322 , which is less than 0.1 . The result is consistent.

Table 4. Weighing of digital exchanges based on the criterion of the lowest amount of commission

\begin{tabular}{|c|c|c|c|c|c|c|c|}
\hline $\begin{array}{c}\text { Based on } \\
\text { the lowest } \\
\text { commission }\end{array}$ & Bybit & Okay X. & Kraken & BitMex & Bittrex & Binance & Coinbase \\
\hline Coinbase & $1 / 5$ & $1 / 2$ & 2 & $1 / 5$ & 1 & $1 / 4$ & $\mathbf{1}$ \\
\hline Binance & $1 / 2$ & 2 & 5 & $1 / 2$ & 4 & 1 & $\mathbf{4}$ \\
\hline Bittrex & $1 / 5$ & $1 / 2$ & 2 & $1 / 5$ & 1 & $1 / 4$ & $\mathbf{1}$ \\
\hline BitMex & 1 & 3 & 6 & 1 & 5 & 2 & $\mathbf{5}$ \\
\hline Kraken & $1 / 6$ & $1 / 4$ & 1 & $1 / 6$ & $1 / 2$ & $1 / 5$ & $\mathbf{1 / 2}$ \\
\hline Okay X. & $1 / 3$ & 1 & 4 & $1 / 3$ & 2 & $1 / 2$ & $\mathbf{2}$ \\
\hline
\end{tabular}




\begin{tabular}{|c|c|c|c|c|c|c|c|}
\hline Bybit & 1 & 3 & 6 & 1 & 5 & 2 & $\mathbf{5}$ \\
\hline Total & 3.4 & 10.25 & 26 & 3.4 & 18.5 & 6.2 & $\mathbf{1 8 . 5}$ \\
\hline
\end{tabular}

Table 5. Matrix of pairwise comparisons of options based on criteria

\begin{tabular}{|c|c|c|c|c|c|c|c|c|}
\hline $\begin{array}{c}\text { Based on the } \\
\text { lowest } \\
\text { commission }\end{array}$ & Total weights & Bybit & Okay X & Kraken & BitMex & Bittrex & Binance & Coinbase \\
\hline Coinbase & 0.06 & 0.06 & 0.05 & 0.08 & 0.06 & 0.05 & 0.04 & $\mathbf{0 . 0 5}$ \\
\hline Binance & 0.18 & 0.15 & 0.20 & 0.19 & 0.15 & 0.22 & 0.16 & $\mathbf{0 . 2 2}$ \\
\hline Bittrex & 0.06 & 0.06 & 0.05 & 0.08 & 0.06 & 0.05 & 0.04 & $\mathbf{0 . 0 5}$ \\
\hline BitMex & 0.28 & 0.29 & 0.29 & 0.23 & 0.29 & 0.27 & 0.32 & $\mathbf{0 . 2 7}$ \\
\hline Kraken & 0.04 & 0.05 & 0.02 & 0.04 & 0.05 & 0.03 & 0.03 & $\mathbf{0 . 0 3}$ \\
\hline Okay X. & 0.11 & 0.10 & 0.10 & 0.15 & 0.10 & 0.11 & 0.08 & $\mathbf{0 . 1 1}$ \\
\hline Bybit & 0.28 & 0.29 & 0.29 & 0.23 & 0.29 & 0.27 & 0.32 & $\mathbf{0 . 2 7}$ \\
\hline
\end{tabular}

Table 6 . Weighing digital exchanges by criteria based on maximum security

\begin{tabular}{|c|c|c|c|c|c|c|c|}
\hline $\begin{array}{c}\text { Based on maximum } \\
\text { security }\end{array}$ & Bybit & Okay X. & Kraken & BitMex & Bittrex & Binance & Coinbase \\
\hline Coinbase & 5 & 1 & 4 & $1 / 2$ & 3 & 2 & $\mathbf{1}$ \\
\hline Binance & 4 & $1 / 2$ & 3 & $1 / 3$ & 2 & 1 & $\mathbf{1} / \mathbf{2}$ \\
\hline Bittrex & 3 & $1 / 3$ & 2 & $1 / 4$ & 1 & $1 / 2$ & $\mathbf{1} / \mathbf{3}$ \\
\hline BitMex & 6 & 2 & 5 & 1 & 4 & 3 & $\mathbf{2}$ \\
\hline Kraken & 2 & $1 / 4$ & 1 & $1 / 5$ & $1 / 2$ & $1 / 3$ & $\mathbf{1} / \mathbf{4}$ \\
\hline Okay X. & 5 & 1 & 4 & $1 / 2$ & 3 & 2 & $\mathbf{1}$ \\
\hline Bybit & 1 & $1 / 5$ & $1 / 2$ & $1 / 6$ & $1 / 3$ & $1 / 4$ & $\mathbf{1 / 5}$ \\
\hline Total & 26 & 5.28 & 19.5 & 2.95 & 13.83 & 9.08 & $\mathbf{5 . 2 8}$ \\
\hline
\end{tabular}

Table 7. Normalized matrix of criteria based on maximum security

\begin{tabular}{|c|c|c|c|c|c|c|c|c|}
\hline $\begin{array}{c}\text { Based on maximum } \\
\text { security }\end{array}$ & $\begin{array}{c}\text { Total } \\
\text { weights }\end{array}$ & Bybit & $\begin{array}{c}\text { Okay } \\
\text { X. }\end{array}$ & Kraken & BitMex & Bittrex & Binance & Coinbase \\
\hline Coinbase & 0.20 & 0.19 & 0.19 & 0.21 & 0.17 & 0.22 & 0.22 & $\mathbf{0 . 1 9}$ \\
\hline Binance & 0.12 & 0.15 & 0.09 & 0.15 & 0.11 & 0.14 & 0.11 & $\mathbf{0 . 0 9}$ \\
\hline Bittrex & 0.08 & 0.12 & 0.06 & 0.10 & 0.08 & 0.07 & 0.06 & $\mathbf{0 . 0 6}$ \\
\hline BitMex & 0.31 & 0.23 & 0.38 & 0.26 & 0.34 & 0.29 & 0.33 & $\mathbf{0 . 3 8}$ \\
\hline Kraken & 0.05 & 0.08 & 0.05 & 0.05 & 0.07 & 0.04 & 0.04 & $\mathbf{0 . 0 5}$ \\
\hline Okay X. & 0.20 & 0.19 & 0.19 & 0.21 & 0.17 & 0.22 & 0.22 & $\mathbf{0 . 1 9}$ \\
\hline Bybit & 0.04 & 0.04 & 0.04 & 0.03 & 0.06 & 0.02 & 0.03 & $\mathbf{0 . 0 4}$ \\
\hline
\end{tabular}

Table 8 . Weighting of digital exchanges by criteria based on the highest currency code

\begin{tabular}{|c|c|c|c|c|c|c|c|}
\hline $\begin{array}{c}\text { Based on most } \\
\text { currency codes }\end{array}$ & Bybit & Okay $\boldsymbol{X}$. & Kraken & BitMex & Bittrex & Binance & Coinbase \\
\hline Coinbase & 2 & $1 / 2$ & 3 & 1 & $1 / 3$ & $1 / 4$ & $\mathbf{1}$ \\
\hline Binance & 5 & 3 & 6 & 4 & 2 & 1 & $\mathbf{4}$ \\
\hline Bittrex & 4 & 2 & 5 & 3 & 1 & $1 / 2$ & $\mathbf{3}$ \\
\hline BitMex & 2 & $1 / 2$ & 3 & 1 & $1 / 3$ & $1 / 4$ & $\mathbf{1}$ \\
\hline Kraken & $1 / 2$ & $1 / 4$ & 1 & $1 / 3$ & $1 / 5$ & $1 / 6$ & $\mathbf{1 / 3}$ \\
\hline
\end{tabular}




\begin{tabular}{|c|c|c|c|c|c|c|c|}
\hline Okay X. & 3 & 1 & 4 & 2 & $1 / 2$ & $1 / 3$ & $\mathbf{2}$ \\
\hline Bybit & 1 & $1 / 3$ & 2 & $1 / 2$ & $1 / 4$ & $1 / 5$ & $\mathbf{1 / 2}$ \\
\hline Coinbase & 17.5 & 7.58 & 24 & 11.83 & 4.62 & 2.70 & $\mathbf{1 1 . 8 3}$ \\
\hline
\end{tabular}

Table 9. Normalized matrix of criteria based on the highest currency code

\begin{tabular}{|c|c|c|c|c|c|c|c|c|}
\hline $\begin{array}{c}\text { Based on most } \\
\text { currency codes }\end{array}$ & $\begin{array}{c}\text { Total } \\
\text { weights }\end{array}$ & Bybit & $\begin{array}{c}\text { Okay } \\
\text { X. }\end{array}$ & Kraken & BitMex & Bittrex & Binance & Coinbase \\
\hline Coinbase & 0.09 & 0.11 & 0.07 & 0.13 & 0.08 & 0.07 & 0.09 & $\mathbf{0 . 0 8}$ \\
\hline Binance & 0.34 & 0.29 & 0.40 & 0.25 & 0.34 & 0.43 & 0.37 & $\mathbf{0 . 3 4}$ \\
\hline Bittrex & 0.23 & 0.23 & 0.26 & 0.21 & 0.25 & 0.22 & 0.19 & $\mathbf{0 . 2 5}$ \\
\hline BitMex & 0.09 & 0.11 & 0.07 & 0.13 & 0.08 & 0.07 & 0.09 & $\mathbf{0 . 0 8}$ \\
\hline Kraken & 0.04 & 0.03 & 0.03 & 0.04 & 0.03 & 0.04 & 0.06 & $\mathbf{0 . 0 3}$ \\
\hline Okay X. & 0.15 & 0.17 & 0.13 & 0.17 & 0.17 & 0.11 & 0.12 & $\mathbf{0 . 1 7}$ \\
\hline Bybit & 0.06 & 0.06 & 0.04 & 0.08 & 0.04 & 0.05 & 0.07 & $\mathbf{0 . 0 4}$ \\
\hline
\end{tabular}

Table 10. Weighing of digital exchanges by criteria based on support speed

\begin{tabular}{|c|c|c|c|c|c|c|c|}
\hline $\begin{array}{c}\text { Based on support } \\
\text { speed }\end{array}$ & Bybit & Okay $\boldsymbol{X}$. & Kraken & BitMex & Bittrex & Binance & Coinbase \\
\hline Coinbase & $1 / 5$ & $1 / 2$ & 2 & $1 / 3$ & 1 & $1 / 4$ & $\mathbf{1}$ \\
\hline Binance & $1 / 2$ & 3 & 5 & 2 & 4 & 1 & $\mathbf{4}$ \\
\hline Bittrex & $1 / 5$ & $1 / 2$ & 2 & $1 / 3$ & 1 & $1 / 4$ & $\mathbf{1}$ \\
\hline BitMex & $1 / 3$ & 2 & 4 & 1 & 3 & $1 / 2$ & $\mathbf{3}$ \\
\hline Kraken & $1 / 6$ & $1 / 3$ & 1 & $1 / 4$ & $1 / 2$ & $1 / 5$ & $\mathbf{1 / 2}$ \\
\hline Okay X. & $1 / 4$ & 1 & 3 & $1 / 2$ & 2 & $1 / 3$ & $\mathbf{2}$ \\
\hline Bybit & 1 & 4 & 6 & 3 & 5 & 2 & $\mathbf{5}$ \\
\hline Total & 2.65 & 11.33 & 23 & 7.42 & 16.5 & 4.53 & $\mathbf{1 6 . 5}$ \\
\hline
\end{tabular}

Table 11. Normalized matrix of criteria based on support speed

\begin{tabular}{|c|c|c|c|c|c|c|c|c|}
\hline $\begin{array}{c}\text { Based on support } \\
\text { speed }\end{array}$ & $\begin{array}{c}\text { Total } \\
\text { weights }\end{array}$ & Bybit & $\begin{array}{c}\text { Okay } \\
\boldsymbol{X}\end{array}$ & Kraken & BitMex & Bittrex & Binance & Coinbase \\
\hline Coinbase & 0.06 & 0.08 & 0.04 & 0.09 & 0.04 & 0.06 & 0.06 & $\mathbf{0 . 0 6}$ \\
\hline Binance & 0.24 & 0.19 & 0.26 & 0.22 & 0.27 & 0.24 & 0.22 & $\mathbf{0 . 2 4}$ \\
\hline Bittrex & 0.06 & 0.08 & 0.04 & 0.09 & 0.04 & 0.06 & 0.06 & $\mathbf{0 . 0 6}$ \\
\hline BitMex & 0.15 & 0.13 & 0.18 & 0.17 & 0.13 & 0.18 & 0.11 & $\mathbf{0 . 1 8}$ \\
\hline Kraken & 0.04 & 0.06 & 0.03 & 0.04 & 0.03 & 0.03 & 0.04 & $\mathbf{0 . 0 3}$ \\
\hline Okay X. & 0.10 & 0.09 & 0.09 & 0.13 & 0.07 & 0.12 & 0.07 & $\mathbf{0 . 1 2}$ \\
\hline Bybit & 0.35 & 0.38 & 0.35 & 0.26 & 0.40 & 0.30 & 0.44 & $\mathbf{0 . 3 0}$ \\
\hline
\end{tabular}

Table 12. Weighting of digital exchanges by criteria based on trading volume

\begin{tabular}{|c|c|c|c|c|c|c|c|}
\hline $\begin{array}{c}\text { Based on trading } \\
\text { volume }\end{array}$ & Bybit & Okay X & Kraken & BitMex & Bittrex & Binance & Coinbase \\
\hline Coinbase & $1 / 3$ & $1 / 4$ & 3 & $1 / 2$ & 2 & $1 / 5$ & $\mathbf{1}$ \\
\hline Binance & 3 & 2 & 7 & 4 & 6 & 1 & $\mathbf{5}$ \\
\hline Bittrex & $1 / 4$ & $1 / 5$ & 2 & $1 / 3$ & 1 & $1 / 6$ & $\mathbf{1} / \mathbf{2}$ \\
\hline BitMex & $1 / 2$ & $1 / 3$ & 4 & 1 & 3 & $1 / 4$ & $\mathbf{2}$ \\
\hline Kraken & $1 / 5$ & $1 / 6$ & 1 & $1 / 4$ & $1 / 2$ & $1 / 7$ & $\mathbf{1} / \mathbf{3}$ \\
\hline
\end{tabular}




\begin{tabular}{|c|c|c|c|c|c|c|c|}
\hline Okay X. & 2 & 1 & 6 & 3 & 5 & $1 / 2$ & $\mathbf{4}$ \\
\hline Bybit & 1 & $1 / 2$ & 5 & 2 & 4 & $1 / 3$ & $\mathbf{3}$ \\
\hline Total & 7.28 & 4.45 & 28 & 11.08 & 21.50 & 2.59 & $\mathbf{1 5 . 8 3}$ \\
\hline
\end{tabular}

Table 13. Normalized matrix of criteria based on trading volume

\begin{tabular}{|c|c|c|c|c|c|c|c|c|}
\hline $\begin{array}{c}\text { Based on } \\
\text { trading volume }\end{array}$ & $\begin{array}{c}\text { Total } \\
\text { weights }\end{array}$ & Bybit & $\begin{array}{c}\text { Okay } \\
\text { X. }\end{array}$ & Kraken & BitMex & Bittrex & Binance & Coinbase \\
\hline Coinbase & 0.07 & 0.05 & 0.06 & 0.11 & 0.05 & 0.09 & 0.08 & $\mathbf{0 . 0 6}$ \\
\hline Binance & 0.35 & 0.41 & 0.45 & 0.25 & 0.36 & 0.28 & 0.39 & $\mathbf{0 . 3 2}$ \\
\hline Bittrex & 0.05 & 0.03 & 0.04 & 0.07 & 0.03 & 0.05 & 0.06 & $\mathbf{0 . 0 3}$ \\
\hline BitMex & 0.11 & 0.07 & 0.07 & 0.14 & 0.09 & 0.14 & 0.10 & $\mathbf{0 . 1 3}$ \\
\hline Kraken & 0.03 & 0.03 & 0.04 & 0.04 & 0.02 & 0.02 & 0.06 & $\mathbf{0 . 0 2}$ \\
\hline Okay X. & 0.24 & 0.27 & 0.22 & 0.21 & 0.27 & 0.23 & 0.19 & $\mathbf{0 . 2 5}$ \\
\hline Bybit & 0.16 & 0.14 & 0.11 & 0.18 & 0.18 & 0.19 & 0.13 & $\mathbf{0 . 1 9}$ \\
\hline
\end{tabular}

Table 14. Easier and better weighting of digital exchanges based on authentication process

\begin{tabular}{|c|c|c|c|c|c|c|c|}
\hline $\begin{array}{c}\text { Easier and better based } \\
\text { on the authentication } \\
\text { process }\end{array}$ & Bybit & $\begin{array}{c}\text { Okay } \\
\mathbf{X} .\end{array}$ & Kraken & BitMex & Bittrex & Binance & Coinbase \\
\hline Coinbase & 3 & $1 / 2$ & 3 & 2 & 2 & $1 / 2$ & $\mathbf{1}$ \\
\hline Binance & 3 & 1 & 3 & 2 & 3 & 1 & $\mathbf{2}$ \\
\hline Bittrex & 2 & $1 / 3$ & 2 & $1 / 2$ & 1 & $1 / 3$ & $\mathbf{1 / 2}$ \\
\hline BitMex & 2 & $1 / 2$ & 3 & 1 & 2 & $1 / 2$ & $\mathbf{1 / 2}$ \\
\hline Kraken & 1 & $1 / 3$ & 1 & $1 / 3$ & $1 / 2$ & $1 / 3$ & $\mathbf{1 / 3}$ \\
\hline Okay X. & 3 & 1 & 3 & 2 & 3 & 1 & $\mathbf{2}$ \\
\hline Bybit & 1 & $1 / 3$ & 1 & $1 / 2$ & $1 / 2$ & $1 / 3$ & $\mathbf{1 / 3}$ \\
\hline Total & 15 & 4 & 16 & 8.33 & 12 & 4 & $\mathbf{6 . 8 3}$ \\
\hline
\end{tabular}

Table 15. Normalized matrix of criteria based on easier and better authentication process

\begin{tabular}{|c|c|c|c|c|c|c|c|c|}
\hline $\begin{array}{c}\text { Easier and better } \\
\text { based on the } \\
\text { authentication } \\
\text { process }\end{array}$ & $\begin{array}{c}\text { Total } \\
\text { weights }\end{array}$ & Bybit & $\begin{array}{c}\text { Okay } \\
\text { X. }\end{array}$ & Kraken & BitMex & Bittrex & Binance & Coinbase \\
\hline Coinbase & 0.17 & 0.20 & 0.13 & 0.19 & 0.24 & 0.17 & 0.13 & $\mathbf{0 . 1 5}$ \\
\hline Binance & 0.24 & 0.20 & 0.25 & 0.19 & 0.24 & 0.25 & 0.25 & $\mathbf{0 . 2 9}$ \\
\hline Bittrex & 0.09 & 0.13 & 0.08 & 0.13 & 0.06 & 0.08 & 0.08 & $\mathbf{0 . 0 7}$ \\
\hline BitMex & 0.13 & 0.13 & 0.13 & 0.19 & 0.12 & 0.17 & 0.13 & $\mathbf{0 . 0 7}$ \\
\hline Kraken & 0.06 & 0.07 & 0.08 & 0.06 & 0.04 & 0.04 & 0.08 & $\mathbf{0 . 0 5}$ \\
\hline Okay X. & 0.24 & 0.20 & 0.25 & 0.19 & 0.24 & 0.25 & 0.25 & $\mathbf{0 . 2 9}$ \\
\hline Bybit & 0.07 & 0.07 & 0.08 & 0.06 & 0.06 & 0.04 & 0.08 & $\mathbf{0 . 0 7}$ \\
\hline
\end{tabular}




\section{Conclusion}

This research aimed to analyze and select the best exchange in digital currencies with a futuristic perspective. Through surveys conducted first, while introducing the top 7 exchange offices in digital currencies, the strengths and weaknesses of each were examined. In the next step, the most important indicators and criteria in selecting the best exchange in the field of services were extracted. By weighting and normalizing the criteria matrix through hierarchical analysis (AHP), each of these exchanges was examined and prioritized in the specified criteria. The most important criteria in choosing the best digital currency exchange are: security, support, commission, number of currency codes, authentication and trading volume. Finally, the results of this research can be used for future research and investment in the field of cryptocurrencies.

\section{Suggestion for future research}

As a suggestion for future research, it is possible to formulate the framework required for accounting according to the Fourth Industrial Revolution by studying it more broadly and improving its validity and reliability by examining the infrastructure of various countries. It also compared exchange offices providing services related to cryptocurrencies or digital currencies with other methods and compared the results with the results of this study.

\section{References}

Aaron, M., Rivadeneyra, F., \& Sohal, S. (2017). Fintech: is this time different? a framework for assessing risks and opportunities for central banks. Retrieved from.

Alving, A. S., Craige, B., Jones, R., Whorton, C. M., Pullman, T. N., \& Eichelberger, L. (1948). Pentaquine (sn-13,276), a therapeutic agent effective in reducing the relapse rate in viv ax malaria. The Journal of clinical investigation, 27(3), 25-33.

Allayannis, G., Ihrig, J., \& Weston, J. P. (2001). Exchange-rate hedging: Financial versus operational strategies. American Economic Review, 91(2), 391-395.

Anderson, R., Shumailov, I., Ahmed, M., \& Rietmann, A. (2019). Bitcoin redux.

Batent, C., Taheri, M., Borbor, M., \& Nasri, S. (2020). Cytotoxic effects of methanolic extract of ziziphora tenuior 1 . on the growth of the lung cancer cell line. FABAD Journal of Pharmaceutical Sciences, 45(3), 195-204.

Brunton, S. L., Noack, B. R., \& Koumoutsakos, P. (2020). Machine learning for fluid mechanics. Annual Review of Fluid Mechanics, 52, 477-508.

Dell'Erba, M. (2019). Stablecoins in cryptoeconomics from initial coin offerings to central bank digital currencies. NYUJ Legis. \& Pub. Pol'y, 22, 1.

Di Silvestre, M. L., Gallo, P., Guerrero, J. M., Musca, R., Sanseverino, E. R., Sciumè, G., ... \& Zizzo, G. (2020). Blockchain for power systems: Current trends and future applications. Renewable and Sustainable Energy Reviews, 119, 109585.

Ding, Y.-J., Li, T., Hong, X.-J., Zhu, L.-C., Cai, Y.-P., Zhu, S.-M., \& Yu, S.-J. (2015). Construction of four $3 \mathrm{~d}-4 \mathrm{f}$ heterometallic pillar-layered frameworks containing left-and right-handed helical chains and a I- chemosensor. CrystEngComm, 17(21), 3945-3952.

Foroglou, G., \& Tsilidou, A.-L. (2015). Further applications of the blockchain. Paper presented at the 12th student conference on managerial science and technology.

Go, A. S., Mozaffarian, D., Roger, V. L., Benjamin, E. J., Berry, J. D., Blaha, M. J., . . Franco, S. (2014). Heart disease and stroke statistics - 2014 update: a report from the American Heart Association. Circulation, 129(3), e28-e292.

Hakimi-Asiabar, M., Ghodsipour, S., Seifi, A., Kerachian, R., \& O'Brien, C. (2008). A multi-objective hybrid gradient-based genetic algorithm. Paper presented at the Fifteenth international working seminar on production economics, Innsbruck, Austria.

Howell, S. T., Niessner, M., \& Yermack, D. (2020). Initial coin offerings: Financing growth with cryptocurrency token sales. The Review of Financial Studies, 33(9), 3925-3974.

Irresberger, F., John, K., \& Saleh, F. (2020). The public blockchain ecosystem: An empirical analysis. NYU Stern School of Business.

Khaleghi Moghadam, H., \& Khalegh, A. (2009). Corporate transparency in Iran and effective factors on them. UCT Journal, 6(21), 31-60. 
Mainelli, M., \& Smith, M. (2015). Sharing ledgers for sharing economies: an exploration of mutual distributed ledgers (aka blockchain technology). Journal of financial perspectives, 3(3).

Mokhtarian, E., \& Lindgren, A. (2018). Rise of the Crypto Hedge Fund: Operational Issues and Best Practices for an Emergent Investment Industry. Stan. JL Bus. \& Fin., 23, 112.

Lei, J., Sun, L., Huang, S., Zhu, C., Li, P., He, J., ... \& He, Q. (2019). The antimicrobial peptides and their potential clinical applications. American journal of translational research, 11(7), 3919.

Picard, C., Al-Herz, W., Bousfiha, A., Casanova, J.-L., Chatila, T., Conley, M. E., . . Klein, C. (2015). Primary immunodeficiency diseases: an update on the classification from the International Union of Immunological Societies Expert Committee for Primary Immunodeficiency 2015. Journal of clinical immunology, 35(8), 696-726.

Pichler, P., Summer, M., \& Weber, B. (2020). Does digitalization require Central Bank Digital Currencies for the general public?. Monetary Policy \& the Economy, 4, 40-56.

Plesner, U., \& Husted, E. (2020). Digital organizing: Revisiting themes in organization studies: Red Globe Press.

Saati, S. M., Memariani, A., \& Jahanshahloo, G. R. (2002). Efficiency analysis and ranking of DMUs with fuzzy data. Fuzzy Optimization and Decision Making, 1(3), 255-267.

Salganik, M. J. (2019). Bit by bit: Social research in the digital age: Princeton University Press.

Savelyev, A. (2017). Contract law 2.0: 'Smart'contracts as the beginning of the end of classic contract law. Information \& Communications Technology Law, 26(2), 116-134.

Tahriri, F., Osman, M. R., Ali, A., Yusuff, R., \& Esfandiary, A. (2008). AHP approach for supplier evaluation and selection in a steel manufacturing company. Journal of Industrial Engineering and Management (JIEM), 1(2), 54-76.

Zahedi, M. R., \& Khanachah, S. N. (2020). The effect of knowledge management processes on organizational innovation through intellectual capital development in Iranian industrial organizations. Journal of Science and Technology Policy Management. 